Article

\title{
Symmetric Informationally-Complete Quantum States as Analogues to Orthonormal Bases and Minimum-Uncertainty States
}

\author{
D. Marcus Appleby ${ }^{1,}$ Hoan Bui Dang ${ }^{2, *}$ and Christopher A. Fuchs ${ }^{3}$ \\ ${ }^{1}$ School of Physics, University of Sydney, Sydney, NSW 2006, Australia; \\ E-Mail: marcus.appleby@ gmail.com \\ ${ }^{2}$ University of Waterloo, 200 University Ave West, Waterloo, ON N2L 3G1, Canada \\ ${ }^{3}$ Quantum Information Processing Group, Raytheon BBN Technologies, 10 Moulton Street, \\ Cambridge, MA 02138, USA; E-Mail: qbism.fuchs@gmail.com \\ * Author to whom correspondence should be addressed; E-Mail: hdang@uwaterloo.ca; \\ Tel.:1-519-569-7600.
}

Received: 21 January 2014; in revised form: 4 February 2014 / Accepted: 5 March 2014 /

Published: 14 March 2014

\begin{abstract}
Recently there has been much effort in the quantum information community to prove (or disprove) the existence of symmetric informationally complete (SIC) sets of quantum states in arbitrary finite dimension. This paper strengthens the urgency of this question by showing that if SIC-sets exist: (1) by a natural measure of orthonormality, they are as close to being an orthonormal basis for the space of density operators as possible; and (2) in prime dimensions, the standard construction for complete sets of mutually unbiased bases and Weyl-Heisenberg covariant SIC-sets are intimately related: The latter represent minimum uncertainty states for the former in the sense of Wootters and Sussman. Finally, we contribute to the question of existence by conjecturing a quadratic redundancy in the equations for Weyl-Heisenberg SIC-sets.
\end{abstract}

Keywords: SIC-POVMs; minimum-uncertainty states

\section{Introduction}

Recently, there has been significant interest in the quantum-information community to prove or disprove the general existence of so-called symmetric informationally complete (SIC) quantum 
measurements [1-10]. The question is simple enough: For a Hilbert space $\mathcal{H}_{d}$ of arbitrary dimension $d$, is there always a set of $d^{2}$ quantum states $\left|\psi_{i}\right\rangle$ such that $\left|\left\langle\psi_{i} \mid \psi_{j}\right\rangle\right|^{2}=$ constant for all $i \neq j$ ? If so, then the operators $E_{i}=\frac{1}{d}\left|\psi_{i}\right\rangle\left\langle\psi_{i}\right|$ can be shown to form the elements of a tomographically complete positive operator-valued measure, often dubbed a SIC-POVM. Interestingly, despite the elementary feel to this question-i.e., it seems the sort of thing one might find as an exercise in a linear-algebra textbook-and the considerable efforts to solve it, the answer remains elusive. One should ask: why is there such interest in the question in the first place? Unfortunately it is hard to deny the impression that much of the motivation stems from little more than the mathematical challenge-not really a good physical reason for months of effort. In this paper, we address the issue of whether there are other, more physical motivations for seeking an answer to the existence of such states.

Indeed, there are already notable motivations from applied physics-e.g., uses of these states in quantum cryptography [11-14]. Here, however, we go further by deriving a few previously unobserved properties that single out SIC-sets as particularly relevant to the elementary structure of Hilbert space. These properties make no direct use of the original defining symmetry for SICs, and instead imply them. It is our hope that a better understanding of SIC-sets will lead to new and powerful tool for studying the structure of quantum mechanics [15-17].

Specifically, the paper is as follows. We first demonstrate a geometric sense in which SIC-sets of projectors $\Pi_{i}=\left|\psi_{i}\right\rangle\left\langle\psi_{i}\right|$, if they exist, are as close as possible to being an orthonormal basis on the cone of nonnegative operators. This complements the frame theoretic version of the same question proved by Scott [18]. The two results together clinch the idea that SIC-sets are promising candidates for a stand-in for orthonormal bases on the space of density operators, and we take the opportunity to express the structure of pure states with respect to these preferred bases. Thereafter, we focus on the case of Weyl-Heisenberg (WH) covariant SIC-sets in prime dimensions. In prime dimensions, complete sets of mutually unbiased bases always exist [19] and here we demonstrate a simple expression for them in terms of the WH unitary operators. We then define a notion of minimum uncertainty state with respect to the measurement of these bases [20] and find that the Weyl-Heisenberg SIC-sets, whenever they exist, consist solely of minimum uncertainty states. This provides a strong motivation for considering WH SIC-sets as a special finite-dimensional analogue of coherent states and stresses the further interest of writing the quantum mechanical state space in terms of such operator bases. Finally, we conclude with some general remarks and conjecture a significant reduction in the defining equations for WH SIC-sets.

\section{Quasi-Orthonormal Bases and the Space of Density Operators}

When equipped with the usual Hilbert-Schmidt inner product $\langle A, B\rangle=\operatorname{tr}\left(A^{\dagger} B\right)$, the set of operators acting on a $d$-dimensional complex vector space becomes a $d^{2}$-dimensional Hilbert space. Suppose we are given $d^{2}$ operators $A_{i}$ normalized so that $\operatorname{tr}\left(A_{i}^{\dagger} A_{i}\right)=1$. Under what conditions can the $A_{i}$ be orthogonal to each other? For instance, it is known that one can require the $A_{i}$ to be Hermitian or unitary and it is not too restrictive for orthogonality to obtain [21]. However, requiring the operators to be positive semi-definite is another matter. 
We would like to measure "how orthonormal" a set of positive semi-definite $A_{i}$ can be. A natural class of measures for this is

$$
K_{t}=\sum_{i \neq j}|\langle A, B\rangle|^{t}=\sum_{i \neq j}\left(\operatorname{tr}\left(A_{i} A_{j}\right)\right)^{t}
$$

for any real number $t \geq 1$. Clearly, one will have an orthonormal basis if and only if $K_{t}=0$ for this sum of $N=d^{4}-d^{2}$ terms. But for positive semi-definite $A_{i}$, as we will prove, it turns out that

$$
K_{t} \geq \frac{d^{2}(d-1)}{(d+1)^{t-1}}
$$

In other words, it is impossible to choose an orthonormal basis all of whose elements lie in the positive cone of operators. The natural question to ask is, is there a set of $d^{2} A_{i}$ for which $K_{t}$ achieves the lower bound for $t>1$ (for $t=1$, any set of $A_{i} / d$ that forms a POVM would saturate the bound for $K_{1}$ )? If so, we will refer to such a set as a quasi-orthonormal basis.

Note that the linear independence of the $A_{i}$ does not have to be imposed as a separate requirement. An orthonormal set of vectors is automatically linearly independent. Similarly with quasi-orthonormal sets: requiring $K_{t}$ to achieve its lower bound forces linear independence. This will follow from one condition for achieving equality—-that $\left\langle A_{i}, A_{j}\right\rangle=$ constant when $i \neq j$ [2].

We will first prove Equation (2) for the case $t=1$, using a special instance of the Cauchy-Schwarz inequality: namely, if $\lambda_{r}$ is any set of $n$ real numbers, $n \sum_{r} \lambda_{r}^{2}=\left(\sum_{r} 1^{2}\right)\left(\sum_{r} \lambda_{r}^{2}\right) \geq\left(\sum_{r} \lambda_{r}\right)^{2}$, with equality if and only if $\lambda_{1}=\cdots=\lambda_{n}$. Let $G$ be a positive semi-definite operator defined by $G=\sum_{i} A_{i}$, and note that $\operatorname{tr} A_{i} \geq 1$ because $\operatorname{tr} A_{i}^{2}=1$. Applying the Schwarz inequality to the eigenvalues of $G$, we find $\operatorname{tr} G^{2} \geq \frac{1}{d}(\operatorname{tr} G)^{2} \geq d^{3}$, or equivalently, $K_{1} \geq d^{3}-d^{2}$. Equality is obtained if and only if $G=d I$ and $\operatorname{tr} A_{i}=1$ for all $i$, i.e., $A_{i}$ are all rank-1 projectors.

Next, let $f(x)=x^{t}$, then $f(x)$ is a strictly convex function for $t>1$. By writing $K_{t}=\sum_{i \neq j} f\left(\operatorname{tr}\left(A_{i} A_{j}\right)\right)$, and applying Jensen inequality, we complete the proof for Equation (2). In Jensen inequality, the equality holds if and only if $\operatorname{tr}\left(A_{i} A_{j}\right)$ is a constant $c$ for all $i \neq j$. One can determine this constant $c$ by taking the trace of both sides of the equation $\left(\sum A_{i}\right)^{2}=G^{2}=d^{2} I$, which comes from the equality condition for the previous Cauchy-Schwarz inequality, to get $c=\left(d^{3}-\sum \operatorname{tr} A_{i}^{2}\right) /\left(d^{4}-d^{2}\right)=1 /(d+1)$.

Putting all this together, we conclude that the necessary and sufficient conditions for the $A_{i}$ to constitute a quasi-orthonormal basis are:

(1) Each $A_{i}$ is a rank-1 projector.

(2) $\operatorname{tr}\left(A_{i} A_{j}\right)=\frac{1}{d+1}$ for all $i \neq j$.

These are the same as the conditions for the operators $A_{i}$ to constitute a SIC-set. In particular they imply $\sum_{i} A_{i}=d I$ and, via [2], that the $A_{i}$ must be linearly independent.

As an aside, let us point out that this derivation sheds light on the meaning of the "second frame potential" $\Phi=\sum_{i, j}\left|\left\langle\psi_{i} \mid \psi_{j}\right\rangle\right|^{4}$ introduced by Renes et al. [3] to aid in finding SIC-POVMs numerically. Beforehand its motivation seemed to be only the heuristic of constrained particles distancing themselves from each other because of an interaction potential—an idea that has its roots in Reference [22]. Now, 
one sees that whenever $A_{i}=\left|\psi_{i}\right\rangle\left\langle\psi_{i}\right|, \Phi=K_{2}+d^{2}$-i.e., $\Phi$ is essentially our own orthonormality measure in this case. Equation (2) implies that $\Phi \geq 2 d^{3} /(d+1)$, which was proved by different means in Reference [3].

Returning to the general development, what we have shown is that SIC-sets $\Pi_{i}=\left|\psi_{i}\right\rangle\left\langle\psi_{i}\right|$, $i=1, \ldots, d^{2}$, play a special role in the geometry of the cone of positive operators, and by implication, the convex set of quantum states in general-i.e., the density operators. For the purpose of foundational studies - particularly ones of the quantum Bayesian variety [15-17,23-25] —it is worthwhile recording what this convex set looks like from this perspective.

Imagine introducing $E_{i}=\frac{1}{d} \Pi_{i}$ as a canonically given quantum measurement. For a quantum state $\rho$, the outcomes will occur with probabilities $p(i)=\operatorname{tr} \rho E_{i}$. Using the fact that $\rho$ has a unique expansion in terms of the $\Pi_{i}$, one can work out that

$$
\rho=\sum_{i}\left((d+1) p(i)-\frac{1}{d}\right) \Pi_{i}
$$

On the other hand, one might imagine taking the vector of probabilities $p(i)$ as the more basic specification, and the density operator $\rho$ as a convenient, but derivative, specification of the quantum state. Requisite to doing this, one must have an understanding of the allowed probabilities $p(i)$-not every choice of probabilities in Equation (3) will give rise to positive semi-definite $\rho$. This can be done conveniently by taking note of the characterization of pure quantum states demonstrated in Reference [26]. Remarkably, it is enough to specify that any Hermitian operator $M$ satisfy only two trace conditions, $\operatorname{tr} M^{2}=1$ and $\operatorname{tr} M^{3}=1$, to insure that it be a rank-1 projection operator-i.e., a pure state. One can show that by letting $r_{i}$ be the real eigenvalues of $M$ and noticing that the first condition implies $\left|r_{i}\right| \leq 1$ for all $i$, and that $\operatorname{tr} M^{2}-\operatorname{tr} M^{3}=\sum_{i} r_{i}^{2}\left(1-r_{i}\right) \geq 0$, with equality if and only if one of the $r_{i}$ is 1 and the rest are 0 . In terms of the $p(i)$ these two conditions become:

$$
\begin{aligned}
\sum_{i} p(i)^{2} & =\frac{2}{d(d+1)} \\
\sum_{i, j, k} c_{i j k} p(i) p(j) p(k) & =\frac{d+7}{(d+1)^{3}}
\end{aligned}
$$

where $c_{i j k}=\operatorname{Re} \operatorname{tr}\left(\Pi_{i} \Pi_{j} \Pi_{k}\right)$. The full set of quantum states is thus the convex hull of the solutions to Equations (4) and (5). One nice thing about Equation (5) is that it makes transparent the algebraic structure lying behind these special probability vectors: For, one can use the coefficients $c_{i j k}$ as structure coefficients in defining the anticommutator on the space of operators.

\section{Weyl-Heisenberg SIC-Sets and Minimum Uncertainty States}

A particularly important class of SIC-sets are those covariant under the action of the Weyl-Heisenberg (WH) group. Most of the SIC-sets constructed to date are of this variety [1,3-8,10], and these structures have many pleasing properties. In this Section, we derive a few new properties of such sets and show a sense in which their elements can be called minimum uncertainty states when $d$ is prime.

Choose a standard basis $|0\rangle, \ldots,|d-1\rangle$ in $\mathcal{H}_{d}$. Let $Z|j\rangle=\omega^{j}|j\rangle$ and $X|j\rangle=|j+1\rangle$, where $\omega=e^{2 \pi i / d}$ and the addition in $|j+1\rangle$ is modulo $d$. The WH displacement operators are defined by 
$D_{\mathbf{r}}=\tau^{r_{1} r_{2}} X^{r_{1}} Z^{r_{2}}$ for $\mathbf{r}=\left(r_{1}, r_{2}\right)$ and $\tau=-e^{i \pi / d}$. A WH covariant SIC-set is constructed by taking a single normalized vector $|\psi\rangle$ and acting on it with the unitaries $D_{\mathbf{r}}$. As $r_{1}, r_{2}$ range over the integers $0, \ldots, d-1$ this gives $d^{2}$ vectors $\left|\psi_{\mathbf{r}}\right\rangle=D_{\mathbf{r}}|\psi\rangle$. If a $|\psi\rangle$ exists such that

$$
\left\langle\psi\left|D_{\mathbf{r}}\right| \psi\right\rangle= \begin{cases}1 & \mathbf{r}=\mathbf{0} \\ \frac{e^{i \theta_{\mathbf{r}}}}{\sqrt{d+1}} & \mathbf{r} \neq \mathbf{0}\end{cases}
$$

the $\left|\psi_{\mathbf{r}}\right\rangle\left\langle\psi_{\mathbf{r}}\right|$ form a SIC-set and we call $|\psi\rangle$ fiducial.

We can write Equation (6) in a more convenient form by working things out directly in terms of components. We have $\left\langle\psi\left|D_{\mathbf{r}}\right| \psi\right\rangle=\tau^{r_{1} r_{2}} \sum_{j} \omega^{j r_{2}} \psi_{j+r_{1}}^{*} \psi_{j}$ where $\psi_{j}=\langle j \mid \psi\rangle$. Consequently, taking a Fourier transform,

$$
\frac{1}{d} \sum_{r_{2}} \omega^{k r_{2}}\left|\left\langle\psi\left|D_{\mathbf{r}}\right| \psi\right\rangle\right|^{2}=\sum_{j} \psi_{j} \psi_{j+k}^{*} \psi_{j+r_{1}}^{*} \psi_{j+k+r_{1}}
$$

Replacing $\left|\left\langle\psi\left|D_{\mathbf{r}}\right| \psi\right\rangle\right|^{2}$ with $\left(d \delta_{\mathbf{r}, \mathbf{0}}+1\right) /(d+1)$ we deduce that $|\psi\rangle$ is a fiducial vector if and only if

$$
\sum_{j} \psi_{j} \psi_{j+k}^{*} \psi_{j+l}^{*} \psi_{j+k+l}=\frac{1}{d+1}\left(\delta_{k 0}+\delta_{l 0}\right)
$$

In the equations $\left|\left\langle\psi\left|D_{\mathbf{r}}\right| \psi\right\rangle\right|^{2}=\left(d \delta_{\mathbf{r}, \mathbf{0}}+1\right) /(d+1)$, the left hand sides are sums of terms quartic in the components, multiplied by powers of $\omega$. In Equation (8) the powers of $\omega$ have disappeared; this makes them easier to work with. Similarly, note that the frame potential $\Phi$ can be written in an $\omega$-free form. Starting from Equation (7), it follows that

$$
\Phi=d^{3} \sum_{k, l}\left|\sum_{j} \psi_{j} \psi_{j+k}^{*} \psi_{j+l}^{*} \psi_{j+k+l}\right|^{2}
$$

Finally, let us note the important case of Equation (8) where $l=0$. The only terms appearing are then, in terms of probabilities $p_{j}=\left|\psi_{j}\right|^{2}$ :

$$
\sum_{j} p_{j} p_{j+k}=\frac{1}{d+1}\left(1+\delta_{k 0}\right)
$$

Let us now specialize to prime dimension and work toward expressing the fiduciality condition Equation (8) in terms of probabilities of measurement outcomes for a complete set of $d+1$ mutually unbiased bases (MUBs). Assume $d>2$ (the $d=2$ case requires special treatment, though our conclusions continue to hold).

Consider the matrix $F=\left(\begin{array}{ll}\alpha & \beta \\ \gamma & \delta\end{array}\right)$ where $\alpha, \beta, \gamma, \delta$ are nonnegative integers $<d$ such that $\operatorname{det} F=1 \bmod d$. We will refer to such matrices as symplectic matrices. Let $U$ be the unitary

$$
U= \begin{cases}\frac{1}{\sqrt{d}} \sum_{j, k} \tau^{\beta^{-1}\left(\alpha k^{2}-2 j k+\delta j^{2}\right)}|j\rangle\langle k| & \beta \neq 0 \\ \sum_{j} \tau^{\alpha \gamma j^{2}}|\alpha j\rangle\langle j| & \beta=0\end{cases}
$$

Then it can be shown [4] that

$$
U D_{\mathbf{r}} U^{\dagger}=D_{F \mathbf{r}}
$$


for all $\mathbf{r}$. Moreover, if $U^{\prime}$ is any other unitary with this property, then $U^{\prime}=e^{i \phi} U$ for some phase $e^{i \phi}$. In these expressions all arithmetical operations on the indices are $\bmod d . \operatorname{In}$ particular $\beta^{-1}$ is the unique positive integer less than $d$ such that $\beta^{-1} \beta=1 \bmod d$.

If $\beta \neq 0$, it can be seen that $|\langle j|U| k\rangle|=d^{-1 / 2}$ for all $j, k$. So we can use symplectic transformations to generate MUBs. For definiteness take $V, W$ to be the unitaries corresponding to $\left(\begin{array}{ll}1 & 1 \\ 0 & 1\end{array}\right)$ and $\left(\begin{array}{cc}0 & 1 \\ -1 & 0\end{array}\right)$ and define

$$
|m, j\rangle= \begin{cases}V^{m}|j\rangle & m=0,1, \ldots, d-1 \\ W|j\rangle & m=\infty\end{cases}
$$

This gives us a full set of $d+1$ MUBs labeled by $m$.

These bases are essentially the only ones obtainable from the standard basis by symplectic transformations. To see this let $F$ be an arbitrary symplectic matrix with $\beta \neq 0$, and let $U$ be the corresponding unitary. We have

$$
F=\left(\begin{array}{ll}
\alpha & \beta \\
\gamma & \delta
\end{array}\right)= \begin{cases}\left(\begin{array}{cc}
1 & 1 \\
0 & 1
\end{array}\right){ }^{\beta \delta^{-1}}\left(\begin{array}{cc}
\delta^{-1} & 0 \\
\gamma & \delta
\end{array}\right) & \delta \neq 0 \\
\left(\begin{array}{cc}
0 & 1 \\
-1 & 0
\end{array}\right)\left(\begin{array}{cc}
\beta^{-1} & 0 \\
\alpha & \beta
\end{array}\right) & \delta=0\end{cases}
$$

But, unitaries corresponding to matrices with a 0 in the top right-hand position merely permute and re-phase the elements of the standard basis. So the basis $U|0\rangle, \ldots, U|d-1\rangle$ coincides with one of the bases $|m, 0\rangle, \ldots|m,(d-1)\rangle$ up to permutation and re-phasing. We have $|j\rangle\langle j|=(1 / d) \sum_{r} \omega^{-j r} D_{(0, r)}$. In view of Equations (12) and (13) this means

$$
|m, j\rangle\langle m, j|= \begin{cases}\frac{1}{d} \sum_{r} \omega^{-j r} D_{(m r, r)} & m \neq \infty \\ \frac{1}{d} \sum_{r} \omega^{-j r} D_{(r, 0)} & m=\infty\end{cases}
$$

Now consider an arbitrary $|\psi\rangle$, and let $p_{m, j}=|\langle m, j \mid \psi\rangle|^{2}$. It follows from the above that

$$
\sum_{j} p_{m, j} p_{m, j+k}= \begin{cases}\frac{1}{d} \sum_{r} \omega^{k r}\left|\left\langle\psi\left|D_{(m r, r)}\right| \psi\right\rangle\right|^{2} & m \neq \infty \\ \frac{1}{d} \sum_{r} \omega^{k r}\left|\left\langle\psi\left|D_{(r, 0)}\right| \psi\right\rangle\right|^{2} & m=\infty\end{cases}
$$

from which one sees that $|\psi\rangle$ is fiducial if and only if

$$
\sum_{j} p_{m, j} p_{m, j+k}=\frac{1}{d+1}\left(1+\delta_{k 0}\right)
$$

Thus, instead of imposing all of the Equation (8) on the components in the standard basis, we can impose just the special case Equation (10) for each MUB separately.

We are now ready to derive the minimum uncertainty property. Consider an arbitrary state $|\psi\rangle$ and a measurement of one of the bases $m$ in a complete set of MUBs. This will give rise to outcomes with a probability distribution $p_{m, j}$. We will quantify the uncertainty in the outcomes by the quadratic Rényi entropy [20,27]

$$
H_{m}=-\log _{2}\left(\sum_{j} p_{m, j}^{2}\right)
$$


This measure and the measure related to it by deleting the logarithm seem to be playing a widening role in quantum information studies [28]. In particular, it is one of the most important measures for quantifying an eavesdropper's information in quantum cryptography [29].

A minimum uncertainty state is one that minimizes the total uncertainty, $T=\sum_{m} H_{m}$. To see the conditions for this, we first appeal to the fact that $[28,30]$

$$
\sum_{m, j} p_{m, j}^{2}=2
$$

for any $|\psi\rangle$. From the convexity of the logarithm, it follows that for any sequence of positive numbers $\lambda_{1}, \ldots, \lambda_{n},(1 / n) \sum_{j} \log _{2} \lambda_{j} \leq \log _{2}\left((1 / n) \sum_{j} \lambda_{j}\right)$ with equality if and only if $\lambda_{1}=\cdots=\lambda_{n}$. Thus, in view of Equation (19)

$$
T \geq(d+1) \log _{2}\left(\frac{d+1}{2}\right)
$$

with equality if and only if $\sum_{j} p_{m, j}^{2}=2 /(d+1)$ for all $m$. Comparing this with Equation (17), one sees that every WH fiducial vector achieves the lower bound and is therefore a minimum uncertainty state. Unfortunately, the theorem does not go the other way: it is not always the case that every minimum uncertainty state is a fiducial state.

\section{Discussion}

We have argued that the SIC-sets in general dimensions, and more specifically the Weyl-Heisenberg covariant ones (at least in prime dimensions), are particularly interesting structures in Hilbert space geometry. Thus they call out for a better understanding, and we hope this paper is an advertisement for that cause.

There are clearly many more things that can be asked. For instance, are SIC fiducial states also minimum uncertainty states in prime-power dimensions? In non-prime-power dimensions, we face the problem that complete sets of MUBs may not even exist. In that case, can one generalize the definition of a MUB in such a way that SIC fiducial states continue to be minimum uncertainty states? With regard to the property of quasi-orthonormality, is it possible to find non-Weyl-Heisenberg SIC-sets for which Equation (5) takes a nicer form? For instance, if the $\Pi_{i}$ were actually orthogonal, then one would have $\operatorname{tr}\left(\Pi_{i} \Pi_{j} \Pi_{k}\right)=\delta_{i j} \delta_{j k} \delta_{k i}$. How close can one come to a form like this with a SIC-set? Many questions like this loom.

But most looming is the question of whether SIC-sets exist in arbitrary dimension. In this regard, let us close with a conjecture. The necessary and sufficient conditions for $|\psi\rangle$ to be a Weyl-Heisenberg fiducial vector are captured in Equation (8), which represents $d^{2}$ simultaneous equations. However, we have noted numerically (up to $d=28$ ) that satisfying roughly $\frac{3}{2} d$ of these equations is enough to imply the rest. For instance, it appears to be sufficient to satisfy Equation (8) only for the cases $k=0,1,2$ with $l=0, \ldots d / 2$. Perhaps this always holds true. 


\section{Acknowledgments}

This research was supported in part by the U. S. Office of Naval Research (Grant No. N00014-09-1-0247). H. B. Dang is supported by Vanier Canada Graduate Scholarship and Natural Sciences and Engineering Research Council of Canada.

\section{Author Contributions}

All 3 authors were fully involved at all 3 stages of the research: (1) conception and design, (2) drafting and revising, (3) final approval of the version to be published. The work progressed by a process of ongoing discussions and interactions between the authors.

\section{Conflicts of Interest}

The authors declare no conflicts of interest.

\section{References}

1. Zauner, G. Quantum Designs: Foundations of a non-commutative Design Theory. Ph.D. Thesis, University of Vienna, Vienna, Austria, 1999.

2. Caves, C.M. Symmetric informationally complete POVMs Available online: http://info.phys. unm.edu/caves/reports/infopovm.pdf (accessed on 18/11/2013).

3. Renes, J.M.; Blume-Kohout, R.; Scott, A.J.; Caves, C.M. Symmetric informationally complete quantum measurements. J. Math. Phys. 2004, 45, 2171-2180.

4. Appleby, D.M. Symmetric informationally complete positive operator valued measures and the extended Clifford group. J. Math. Phys. 2005, 46, 052107.

5. Grassl, M. On SIC-POVMs and MUBs in dimension 6. 2004, arXiv:quant-ph/0406175.

6. Wootters, W.K. Quantum Measurements and finite geometry. Found. Phys. 2006, 36, 112-126.

7. Klappenecker, A.; Rötteler, M.; Shparlinski, I.E.; Winterhof, A. On approximately symmetric informationally complete positive operator-valued measures and related systems of quantum states. J. Math. Phys. 2005, 46, 082104.

8. Flammia, S.T. On SIC-POVMs in prime dimensions. J. Phys. Math. Gen. 2006, 39, 13483-13493.

9. Scott, A.J.; Grassl, M. Symmetric informationally complete positive-operator-valued measures: A new computer study. J. Math. Phys. 2010, 51, 042203.

10. Appleby, D.M. Symmetric informationally complete measurements of arbitrary rank. Opt. Spectrosc. 2007, 103, 416-428.

11. Fuchs, C.A.; Sasaki, M. Squeezing quantum information through a classical channel: Measuring the "quantumness" of a set of quantum states. Quant. Inform. Comput. 2003, 3, 377-404.

12. Řeháček, J.; Englert, B.G.; Kaszlikowski, D. Minimal qubit tomography. Phys. Rev. A 2004, 70, 052321.

13. Renes, J.M. Equiangular spherical codes in quantum cryptography. Quant. Inform. Comput. 2005, 5, 81-92. 
14. Kim, I.H. Quamtumness, Generalized Spherical 2-Design and Symmetric Informationally Complete POVM. 2007, arXiv:quant-ph/0608024.

15. Fuchs, C.A.; Sasaki, M. On the quantumness of a Hilbert space. Quant. Inform. Comput. 2004, 4, 467-478.

16. Fuchs, C.A. Quantum Mechanics as Quantum Information (and only a little more). 2002, arXiv:quant-ph/0205039.

17. Fuchs, C.A.; Schack, R. Quantum-Bayesian coherence. Rev. Mod. Phys. 2013, 85, 1693-1715.

18. Scott, A.J. Tight informationally complete quantum measurements. J. Phys. Math. Gen. 2006, 39, 13507-13530.

19. Ivonovic, I.D. Geometrical description of quantal state determination. J. Phys. Math. Gen. 1981, $14,3241-3245$.

20. Wootters, W.K.; Sussman, D.M. Discrete phase space and minimum-uncertainty states. 2007, arXiv:0704.1277 [quant-ph].

21. Schwinger, J. Unitary operator bases. Proc. Natl. Acad. Sci. USA 1960, 46, 570-579.

22. Benedetto, J.J.; Fickus, M. Finite Normalized Tight Frames. Adv. Comput. Math. 2003, $18,357-385$.

23. Caves, C.M.; Fuchs, C.A.; Schack, R. Subjective probability and quantum certainty. Stud. Hist. Philos. Mod. Phys. 2007, 38, 255-274.

24. Fuchs, C.A.; Schack, R. Unknown Quantum States and Operations, a Bayesian View; Lecture Notes in Physics; Springer: Berlin, Germany, 2004; p. 147-187.

25. Appleby, D.M. Facts, Values and Quanta. Found. Phys. 2005, 35, 627-668.

26. Jones, N.S.; Linden, N. Parts of quantum states. Phys. Rev. A 2005, 71, 012324.

27. Aczél, J.; Daróczy, Z. On Measures of Information and Their Characterizations; Academic Press: New York, NY, USA 1975; Volume 115.

28. Brukner, C.; Zeilinger, A. Conceptual inadequacy of the Shannon information in quantum measurements. Phys. Rev. A 2001, 63, 022113.

29. Lutkenhaus, N. Estimates for practical quantum cryptography. Phys. Rev. A 1999, doi:10.1103/ PhysRevA.59.3301.

30. Larsen, U. Superspace geometry: The exact uncertainty relationship between complementary aspects. J. Phys. Math Gen. 1990, doi:10.1088/0305-4470/23/7/013.

(c) 2014 by the authors; licensee MDPI, Basel, Switzerland. This article is an open access article distributed under the terms and conditions of the Creative Commons Attribution license (http://creativecommons.org/licenses/by/3.0/). 